\title{
Role of Positron Emission Tomography/Computed Tomography in Evaluation of Hepatic Focal Lesions
}

\author{
MOHAMAD H. EL-SHAFEY, M.D.; MOHAMAD F. SHERIF, M.D.; ALY A. EL-BARBARY, M.D. and \\ MARWA R.E. EL-SAWY, M.Sc.
}

The Department of Radiodiagnosis, Faculty of Medicine, Tanta University, Tanta, Egypt

\begin{abstract}
Background: Positron Emission Tomography/Computed Tomography (PET/CT) has emerged as an increasingly important functional diagnostic tool of various primary or metastatic cancers. PET/CT provides unique information with combined anatomical localization and the metabolic activity of the lesion. In contrast to morphological imaging techniques the ${ }^{18}$ F-flurodeoxyglucose (FDG) PET evaluates tumor viability based on glucose metabolism. By far the most extensive use of PET/CT imaging to date has been in the diagnosis, staging, follow-up and monitoring of malignancies.
\end{abstract}

Aim of Study: The aim of this study was to evaluate the efficiency of PET/CT in the detection and characterization of hepatic focal lesions.

Patients and Methods: Twenty patients were referred with hepatic focal lesions based on previous imaging modalities or histopathological examination who underwent combined PET/CT. Detailed retrograde lesion based and patient based analyses were performed for 84 detected hepatic lesions in 20 patients.

Results: PET/CT showed sensitivity of $92.42 \%$ and specificity of $83.33 \%$ in detection of various hepatic focal lesions and higher sensitivity of $100 \%$ and specificity of $100 \%$ in detection of hepatic metastases from different primaries. Also, PET/CT showed sensitivity of $95.31 \%$ in detection of malignant lesions and sensitivity of $75 \%$ in detection of benign lesions. So, PET/CT was useful to differentiate malignant from benign lesions in the liver.

Conclusion: FDG PET/CT proved to be highly sensitive and specific in the assessment of hepatic focal lesions and was able to differentiate between malignant and benign hepatic focal lesions and their metabolic nature which significantly affect further management.

Key Words: ${ }^{18} F-F D G-P E T / C T-H e p a t i c$ focal lesions.

\section{Introduction}

HEPATIC focal lesions are one of the common annoying radiological findings which have a wide

Correspondence to: Dr. Mohamad H. El-Shafey,

The Department of Radiodiagnosis, Faculty of Medicine, Tanta University, Tanta, Egypt range of pathalogical nature. Hepatocellular Carcinoma (HCC) is the most dangerous and represents the fifth most common malignancy in the world complicating liver cirrhosis in most cases. Its incidence is increasing world wide ranging between $3 \%-9 \%$ annually [1]

The liver is a common repository for many benign and malignant focal lesions. The common benign hepatic focal lesions include hemangioma, focal nodular hyperplasia, cysts and focal fatty change. The primary malignancies that occur in the liver may arise from hepatocytes, bile duct epithelium, endothelial cells, or lymphoid cells, whereas the majority of malignancies encountered in clinical practic are epithelial in origin, with Hepatocellular Carcinoma (HCC) and cholangiocarcinoma accounting for nearly all. The liver is also a very common site for metastases [2,3] .

Recent advances in different imaging techniques increased the ability to detect and characterize hepatic focal lesions, with improvements in diagnosis and monitoring of liver metastases [4].

Unlike conventional imaging, (PET) positron emission tomography combined with (CT) computed tomography (PET/CT) allows an evaluation of the physiological and biochemical processes underlying malignant disease. ${ }^{18} \mathrm{~F}$-FDG PET/CT shows high accuracy and sensitivity in the detection of hepatic metastases derived from a wide range of primary malignant neoplasms. ${ }^{18} \mathrm{~F}$-FDG PET has also been shown to be useful in assessing the chemotherapy treatment response of hepatic metastases $[\mathbf{5 , 6}]$.

Posittron Emission Tomography (PET) is a molecular imaging technique most widely applied in oncology, using ${ }^{18} \mathrm{~F}$ labeled flurodeoxyglucose $\left({ }^{18} \mathrm{~F}-\mathrm{FDG}\right)$. It provides quantitative and qualitative 
functional information about tumor cells depending on their increased rate of glucose metabolism. ${ }^{\mathrm{F}} \mathrm{F}$ FDG PET is regarded to be effective in the detection, staging and restaging of malignancies with a remarkable high sensitivity. The combination of PET and Computed Tomography (CT) represents a very unique imaging modality that scans the whole body in the same session, providing functional and anatomic information in coregistered images. It combines the high sensitivity of PET to the superior anatomical localization by CT [7].

PET/CT hybrid imaging with ${ }^{18} \mathrm{~F}$ fluorodeoxyglucose $\left({ }^{18} \mathrm{~F}-\mathrm{FDG}\right)$ has gained wide application in the diagnosis, staging and follow-up of cancer patients. The degree and amount of ${ }^{18}$ F-FDG uptake in tumor tissues are also valuable indicators in the prognostic stratification of cancer patients [8].

The rationale for the use of the radiotracer FDG for PET/CT imaging in Carcinoma of Unknown Primary (CUP) is the fact that the vast majority of malignant cancer phenotypes exhibit an increased glucose metabolism (Warburg effect). In contrast to CT and conventional MRI, FDG PET/CT offers high lesion-to-background contrast, making it a potentially more sensitive imaging modality for the detection of lesions [9].

The degree of FDG uptake can be expressed quantitatively by means of the Standardized Uptake Value (SUV). It represents the activity in the lesion in $\mathrm{Ci} / \mathrm{nl}$ corrected for the weight of the patient and the dose of FDG administered in which aggressive disease had a high ${ }^{18}$ F-FDG uptake so when SUV is more than 10 it is most likely an aggressive disease [10].

\section{Patients and Methods}

The study was performed in a prospective way carried out in Diagnostic Radiology and Medical Imaging Department at Tanta University Hospitals from October 2016 to October 2017. The study was performed using a PET/CT device. A total number of 20 patients have hepatic focal lesions including 11 males and 9 females. The mean age of the patients was $57 \pm 11$ years (ranged from 29 to 73 years).

\section{Inclusion criteria:}

All of the patients demonstrated blood glucose levels $\leq 150 \mathrm{mg} / \mathrm{dL}$ at the time of injection.

\section{Exclusion criteria:}

- Vital sign instability, severe diabetes, severe illness, active infection.
- Pregnancy.

- Patients with comorbidities, e.g. decompensated cardiovascular or respiratory diseases, unregulated diabetes, acute renal failure or acute infective diseases.

- The study excluded those who had a recent intervention (biopsy), radiotherapy or chemotherapy within 1 month from PET/CT scan.

\section{All patients were asked about:}

Tumor history, received treatment and its duration specially time of last dose, chronic disease, bringing last biopsy histopathological report and serum creatinine level.

The patients were given instruction for the following:

Fasting for $6 \mathrm{~h}$ earlier scan, removal of metallic items and gown wearing. An Intravenous (IV) cannula was inserted for injection of $\left[{ }^{18} \mathrm{~F}\right]$ FDG. height, weight of patients and serum glucose level were routinely measured within 30 minutes before $\left[{ }^{18} \mathrm{~F}\right]$ FDG injection.

The patients were asked to drink 1500c.c. of water to act as neutral oral contrast agent 60 minutes before the examination, and $\left[{ }^{18} \mathrm{~F}\right] \mathrm{FDG}$ was injected manually $60 \mathrm{~min}$ before examination. Patients were asked to rest lonely in a quiet isolated room, devoid of distractions, and they were also asked to keep without movements, including talking to avoid false positive skeletal muscle uptake.

The contrast-enhanced MDCT was performed following injection of $1-2 \mathrm{ml} / \mathrm{Kg}$ of non-ionic contrast medium [ultravist (iopromide)] at a rate of 3$4 \mathrm{ml} / \mathrm{sec}$ by using automatic injector (Medrad Stellant Dual head CT Injector, Germany (for anatomical localization, diagnostic purpose \& attenuation correction errors on the PET images.

\section{Image acquisition and processing:}

All patients underwent PET/CT study using recent hybrid PET/CT system [Philips Medical Systems (Cleveland), Gemini TF (Time-of-Flight) 16 MDCT scanner, USA], consists of a dedicated PET scanner combined with a 16 multi-detector CT scanner. This devoted system permits acquisition of co-recorded CT and PET images in one session. PET/CT scan was performed from skull base to mid-thigh while the patients in supine position using the following parameters: $120 \mathrm{kV}$, $50 \mathrm{~mA}, 5-\mathrm{mm}$ slice thickness, and $0.5 \mathrm{~mm}$ incrimination then PET study were performed. The whole PET/CT study took about $30-45 \mathrm{~min}$. 
PET performed after CT study without any patient movement. Approximately 20 bed positions are planned in 3-D acquisition mode for scanning the same area with 1-2 minutes acquisition time/bed place and there is overlap ( $5 \mathrm{~cm}$ distance) between bed positions for PET/CT scan in caudo-cranial direction.

Reconstruction of spiral PET and CT images were first done then reformatted into coronal and sagittal images to interpret image. Combination of each collected sets of similar PET and CT images done to get fusion images.

Attenuation correction performed by allowing the PET images data reconstruction using CT data then displayed by using dedicated software and elicited on specific workstation.

\section{Images interpretation:}

Every set of images (PET including corrected $\&$ uncorrected images, CT, fused PET/CT images, 3 -D reconstruction) were separately analyzed carefully by at least three radiologists to detect and assess the extent of the $\left[{ }^{18} \mathrm{~F}\right]$ FDG positive true uptake of the disease.

Assessment of PET/CT results as positive or negative was depending upon visual assessment, measuring Standardized Uptake Value (SUV) and comparison of lesions with normal liver background.

\section{Statistical analysis:}

The collected data were statistically analyzed as: Mean \pm Standard Deviation $( \pm S D)$, cases numbers and percentages. Sensitivity, specificity, positive and negative predictive values, and accuracy were calculated to test validity of PET/CT in hepatic focal lesions assessment.

\section{Results}

Twenty patients with 84 hepatic focal lesions confirmed by biopsy or sequential various imaging modalities performed previously, were evaluated for hepatic focal lesions using combined PET/CT scans.

Table (1): Number of cases and percentage of every type of hepatic focal lesions in the 20 patients.

\begin{tabular}{llc}
\hline Hepatic focal lesions & Percentage (\%) & No. of cases \\
\hline Metastases & 40 & 8 \\
HCC & 30 & 6 \\
Lymphoma & 5 & 1 \\
Cholangiocarcinoma & 5 & 1 \\
Dysplastic nodule & 5 & 1 \\
Inflammatory nodule & 5 & 1 \\
Cyst & 5 & 1 \\
Hemangioma & 5 & 1 \\
\hline
\end{tabular}

Role of PET/CT in characterization of lesions: Lesion-based analysis:

Table (2): Comparison of performance indices of CT, PET and PET/CT for characterization of 84 lesions in 20 patients (lesion-based analysis).

\begin{tabular}{lccccc}
\hline & $\begin{array}{c}\text { Sensitivity } \\
(\%)\end{array}$ & $\begin{array}{c}\text { Specificity } \\
(\%)\end{array}$ & $\begin{array}{c}\text { PPV } \\
(\%)\end{array}$ & $\begin{array}{c}\text { NPV } \\
(\%)\end{array}$ & $\begin{array}{c}\text { Accuracy } \\
(\%)\end{array}$ \\
\hline PET/CT & 92.42 & 83.33 & 95.31 & 75 & 90.47 \\
PET & 92.42 & 77.77 & 93.84 & 73.68 & 89.28 \\
CT & 71.21 & 66.66 & 88.67 & 38.70 & 61.28 \\
\hline
\end{tabular}

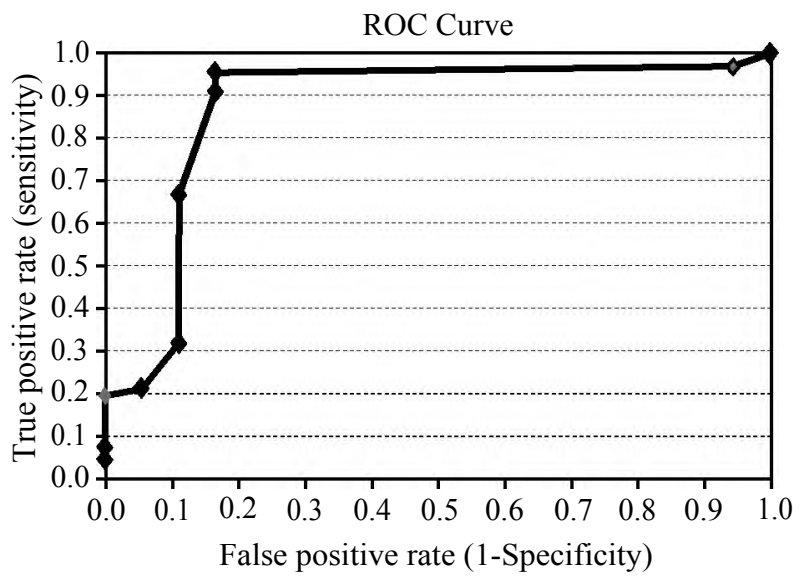

Fig. (1): ROC curves plotted for differentiation between benign and malignant hepatic focal lesions based on SUVmax.

The Receiver Operator Characteristic (ROC) curves of SUVmax in patients with hepatic focal lesions are shown in Fig. (1). ROC analysis showed that the best cut-off value was around 2.5 at our study with sensitivity $96.96 \%$ and specificity $94.44 \%$, SUV max was able to help identify malignant lesions with a higher sensitivity and specificity than benign lesions.

Evaluation of PET/CT diagnostic accuracy: patient-based analysis:

Table (3): Comparison of performance indices of PET/CT, PET and CT based on a patient-based analysis in 20 patients.

\begin{tabular}{llll}
\hline Indices & PET/CT & PET & CT \\
\hline TP & 15 & 15 & 15 \\
TN & 1 & 1 & 3 \\
FP & 2 & 2 & 0 \\
FN & 2 & 2 & 2 \\
Sensitivity (\%) & 88.23 & 88.23 & 88.23 \\
Specificity (\%) & 33.33 & 33.33 & 100 \\
PPV (\%) & 88.23 & 88.23 & 100 \\
NPV (\%) & 33.33 & 33.33 & 60 \\
Accuracy (\%) & 80 & 80 & 90 \\
\hline
\end{tabular}


Table (4): Comparison of performance indices of PET/CT and CT based on a patient-based analysis of hepatic metastases in 20 patients.

\begin{tabular}{|c|c|c|c|c|c|c|c|c|c|c|c|}
\hline & Sensitivity & pecificity & PPV & NPV & Accuracy & & Sensitivity & Specificity & PPV & NPV & Accuracy \\
\hline & & & 11 & & $(95$ & & & & & & $\%)$ \\
\hline PET/CT & $(100 \%)$ & $(100 \%)$ & $(100 \%)$ & $(100 \%)$ & $(100 \%)$ & $\mathrm{PET} /$ & $\Gamma(83.33 \%)$ & $(100 \%)$ & $(100 \%)$ & $(93.33 \%)$ & $(95 \%)$ \\
\hline
\end{tabular}

Case 1:

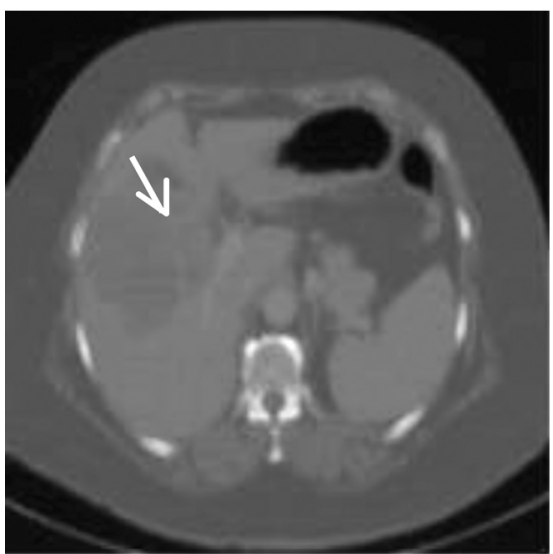

(A)

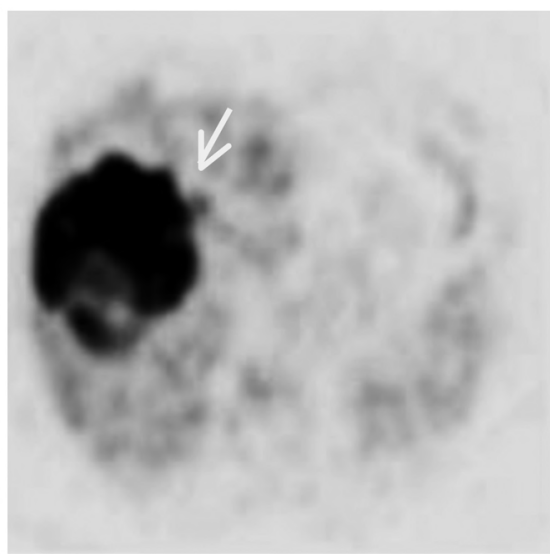

(B)

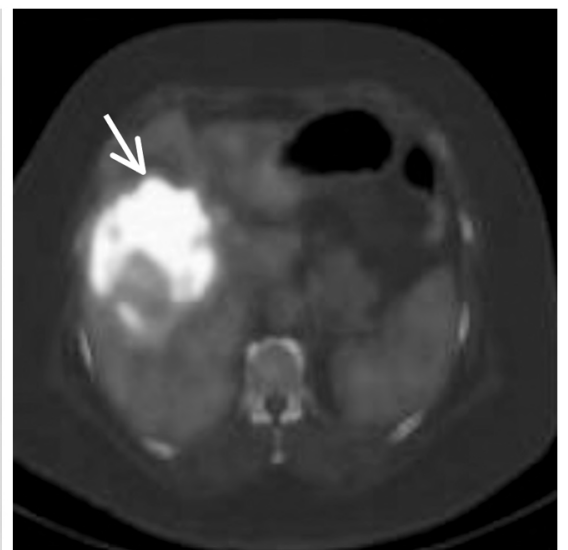

(C)
A 52-year-old female patient with poorly differentiated carcinoma with history of gall bladder carcinoma. (A) Axial multi-slice (16) spiral NECT image showed large well-defined hypodense mass is mainly violating the right hepatic lobe (segment $\mathrm{V}$ ) and gall bladder, corresponding to lesion on CT the axial PET image (B) and axial fused PET/ CT image (C) showed a metabolically active mass displaying eccentric area of breakdown, measuring $8 \times 8.7 \times 10 \mathrm{~cm}$ in maximum dimensions with $\mathrm{SUVmax}=16.5$ (Liver SUVmax=3).

The final diagnosis was a large metastasizing metabolically active malignant hepato-cholecystic mass. These imaging findings are very suggestive for a cholangiocarcinoma.

Case 2:

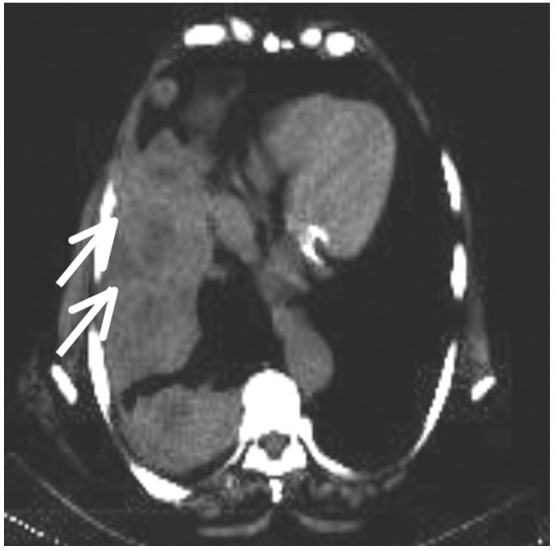

(A)

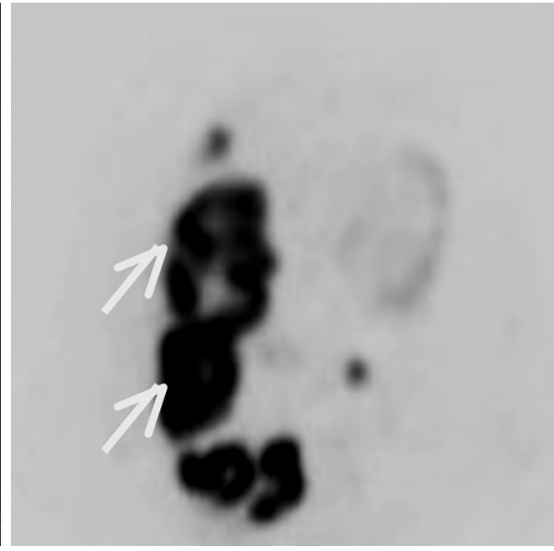

(B)

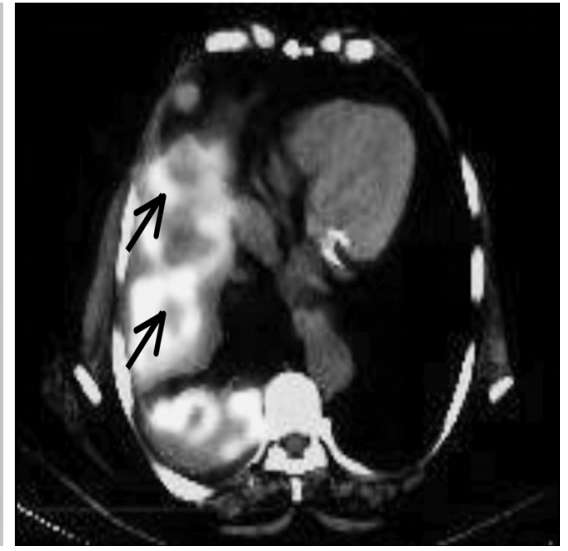

(C)
A 73-year-old female patient with a left breast mass. Axial multi-slice (16) spiral NECT image (A), axial PET image (B), axial fused PET/CT image $(C)$ revealed two space-occupying lesions (SOLs) in segment VII of the right hepatic lobe with the largest one measures about 19 X $13 \mathrm{~mm}$ with $\mathrm{SUV}_{\max }={ }^{9}\left(\mathrm{SUV}_{\max }\right.$ of the liver=3.2).

The final diagnosis was two hepatic metabolically-active deposits. 


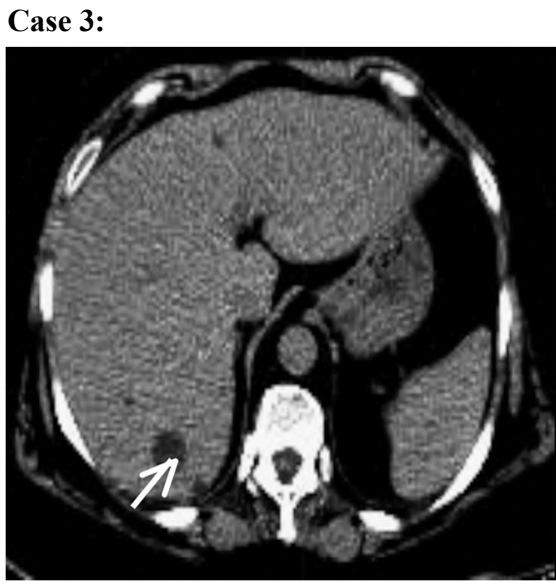

(A)

A 65-year-old female patient with colonic cancer. (A) Axial multi-slice (16) NECT image showed well defined hypodense hepatic focal lesions corresponding lesion (B) axial PET image and (C) fused PET/CT axial image the exhibited metabol-

\section{Discussion}

The distribution of FDG uptake may be useful in characterising the nature of disease. If the increased metabolic activity is generalised or segmental in nature, a diffuse process such as inflammation or infection should be considered, particularly if there are supporting clinical signs or biochemical markers. However there have been reports of primary liver malignancies such as intrahepatic cholangiocarcinoma or lymphoma demonstrating diffuse FDG uptake [11]

Our study demonstrated the FDG PET is not able to discriminate well-differentiated $\mathrm{HCC}$ and dysplastic nodules.

False positive PET diagnoses are related to inflammatory or granulomatous processes. Liver abscess and granulomas can show high FDG uptake and these lesions may be indistinguishable from primary or metastatic liver masses [12]

FDG PET has been shown to be highly sensitive in detecting hepatic metastases from different primaries. Liver metastases are generally FDG avid and, therefore, easily detected by FDG PET. Furthermore, FDG PET has been found to be able to detect extrahepatic metastases that were missed by conventional imaging. This leads to upstaging of patients and a significant change in their management [13] .

PET negative metastases are related to nonFDG avid tumors as bronchioalveolar carcinoma,

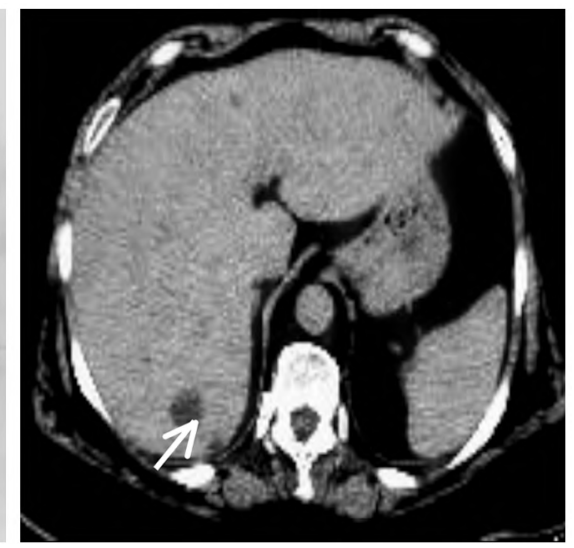

(C)

(B)

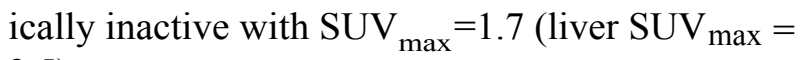
3.5).

The final diagnosis was metabollically inactive hepatic cysts.

carcinoid, neuroendocrine tumors, mucinous-type gastrointestinal cancer, renal and prostatic carcinoma. False negative PET diagnoses can also be related to the presence of lesion too small to be detected or to the prevalence of necrosis and liquefaction respect to tumor tissue [12]

Regarding to patient-based analysis; our study demonstrated higher sensitivity and accuracy of PET/CT over CT in detection of hepatic metastases. CT showed sensitivity of $87.5 \%$, specificity of $100 \%$, Positive Predictive Value (PPV) of 100\%, Negative Predictive Value (NPV) of $92.30 \%$ and accuracy of $95 \%$. However PET/CT showed sensitivity and specificity of $100 \%$ and $100 \%$ respectively with Positive Predictive Value (PPV) of $100 \%$, Negative Predictive Value (NPV) of $100 \%$ and accuracy of $100 \%$.

Our results are agreement with D'Souza et al., (2009) who showed the superiority of PET/CT over CECT in the detection of untreated hepatic metastases in a prospective study evaluating 45 patients with suspected liver metastases from various primary cancers. The sensitivity and specificity in the detection of hepatic metastases were 87.9 and $16.7 \%$, respectively, for CECT, and 97 and 75\%, respectively, for PET/CT [5]

Regarding to patient-based analysis; our study demonstrated higher sensitivity and accuracy of CT over PET/CT in detection of HCC. CT showed sensitivity and specificity of $100 \%$ and $100 \%$ respectively with Positive Predictive Value (PPV) of $100 \%$, Negative Predictive Value (NPV) of 
$100 \%$ and accuracy of $100 \%$. PET/CT showed sensitivity and specificity of $83.33 \%$ and $100 \%$ respectively with Positive Predictive Value (PPV) of $100 \%$, Negative Predictive Value (NPV) of $93.33 \%$ and accuracy of $95 \%$.

Our results are agreement with Tan et al., who showed the superiority of CT over PET/CT in the detection of HCC. PET/CT showed a sensitivity of 55\% to $64 \%$ for hepatocellular carcinoma, compared to $90 \%$ for contrast-enhanced CT [11].

On the other hand this disagree with Niekel et al., (2010) who studied role of PET/CT in detection of HCC in 3391 patients. PET/CT had a higher specificity of $97.2 \%$, compared to $94.9 \%$ for CT [14]

In our study; evaluated one patient with $\mathrm{CC}$, the lesion demonstrated intense FDG uptake.

In the study by Petrowsky et al., PET/CT had no significant advantage over contrast-enhanced $\mathrm{CT}$ in the diagnosis of extrahepatic cholangiocarcinoma as well as intrahepatic lesions [15].

Kim et al., (2008) PET/CT demonstrated no statistically significant advantage over $\mathrm{CT}$ and MR, or MR Cholangiopancreatography (MRCP) in the diagnosis of CC and PET/CT was not superior to $\mathrm{CT}$ or MR/MRCP in detecting primary intrahepatic lesions. Moreover, MR/MRCP showed significantly higher accuracy in the diagnosis of extrahepatic cholangiocarcinoma than PET/CT (92\% versus $80.5 \%$, respectively) [16].

Primary Hepatic Lymphoma (PHL) is extremely rare, presents as a multiple masses as well as diffuse patterns have been described. On CT, it typically presents as a hypoattenuating lesion. A central area of low intensity indicating necrosis may be present. Ring enhancement patterns on dynamic imaging. However, most of these radiological findings are non-specific and such lesions are often misdiagnosed as HCC or as metastases.

Secondary extranodal hepatic lymphoma is more common and PET is used for assessment of treatment response in patients with lymphoma undergoing chemotherapy. Lesions measuring $>1.5 \mathrm{~cm}$ and FDG accumulation exceeding hepatic and splenic FDG are considered positive for lymphoma [13].

Regarding to lesion-based analysis; our study demonstrated higher sensitivity of PET/CT over $\mathrm{CT}$ in detection of hepatic focal lesions. CT showed sensitivity and specificity of $71.21 \%, 66.66 \%$ respectively with Positive Predictive Value (PPV) of $88.67 \%$, Negative Predictive Value (NPV) of $38.70 \%$ and accuracy of $61.28 \%$. However PET/CT showed sensitivity and specificity of $92.42 \%$, $83.33 \%$ respectively with Positive Predictive Value (PPV) of $95.31 \%$, Negative Predictive Value (NPV) of $75 \%$ and accuracy of $90.47 \%$ respectively. Also, $\mathrm{PET} / \mathrm{CT}$ was superior to PET and CT regarding the specificity $(83.33 \%, 77.77 \%$, and $66.66 \%$, respectively).

Another lesion-based analysis on the same study that come in agreement with Ali et al., revealed on the basis of PET/CT a sensitivity, specificity, and accuracy of $100 \%, 89 \%$, and $97 \%$, respectively, and aPV and NPV of $97 \%$ and $100 \%$ and on the basis of PET a sensitivity, specificity, and accuracy of $96 \%, 50 \%$, and $85 \%$, respectively, and a PPV and NPV of $85 \%$ and $82 \%$, respectively [17]

According to Orlacchio et al., the PET study had $94.05 \%$ sensitivity, $91.60 \%$ specificity and 93.36\% accuracy; the CT study had $91.07 \%$ sensitivity, $95.42 \%$ specificity and $92.29 \%$ accuracy. The combined PET/CT had $97.92 \%$ sensitivity, $97.71 \%$ specificity and $97.86 \%$ accuracy. In the present study, according to lesion-based analysis, PET/CT showed sensitivity of $98 \%$, specificity of $100 \%$, PPV of $100 \%$, NPV of $84 \%$ and accuracy of $98 \%$ compared with $98 \%, 98 \%, 99.7 \%, 84 \%$ and $98 \%$, respectively, for PET and sensitivity of $95 \%$, specificity of $81 \%$, PPV of $98 \%$, NPV of $63 \%$ and accuracy of $94 \%$, for CT. So, PET/CT was superior to PET and CT regarding the specificity $(100 \%, 98 \%$, and $81 \%$ respectively) but superior only to $\mathrm{CT}$ regarding the sensitivity $(98 \%$ for PET/CT compared to $95 \%$ for CT) and accuracy (98\% for PET/CT compared to $94 \%$ for CT) [18].

The best cut-off value of SUV $_{\max }$ between the malignant and benign lesions were around 2.5 at our study.

\section{Conclusion:}

FDG-PET/CT proved to be highly sensitive and specific in the assessment of hepatic focal lesions and was able to differentiate between malignant and benign hepatic focal lesions.

In this aspect we had only limitation with patients with low grade (well differentiated) tumors due to the low biological activity of such neoplasms.

FDG-PET/CT proved also to have high sensitivity and specificity in spotting extra hepatic metastatic disease. 
PET/CT is superior to PET and CT alone, in the diagnosis and follow up of various primary or metastatic cancers. Thus, PET/CT is a more accurate test than either of its individual components. PET/CT provides unique information with combined anatomical localization and the metabolic activity of the lesion. In contrast to morphological imaging techniques such as CT, the ${ }^{18}$ F-FDG PET evaluates tumor viability based on glucose metabolism.

\section{References}

1- EL-ZAYADI A., BADRAN H., BARAKAT E., et al.: HCC in Egypt: A single center study over a decade. World Journal of Gastroentrology, 11 (33): 5193-8, 2005.

2- GRAZIOLI L., MORANA G., KIRCHIN M.A., et al.: MRI Contrast for the New Millennium: Contrast-Enhanced MR Imaging of liver masses (CME). Journal of Applied Radiology, 32 (4): 101-26, 2003.

3- MARK H., ROBERT B. and ROBERT M.: Benign liver tumors. Hepatic and biliary disorder. The Merck Manual of diagnosis and Therapy, 302-44, 2005.

4- CHOI J.: Imaging of hepatic metastases. Cancer Control, 13 (1): 6-12, 2006.

5- D'SOUZA M.M., SHARMA R., MONDAL A., et al. Prospective evaluation of CECT and ${ }^{18}$ F-FDG-PET/CT in detection of hepatic metastases. Nucl. Med. Commun., 30 (2): 117-25, 2009.

6- GARCEA G., ONG S.L. and MADDERN G.J.: The current role of PET-CT in the characterization of hepatobiliary malignancies. H.P.B. (Oxford), 11 (1): 4-17, 2009.

7- POEPPEL T., KRAUSE B., HEUSNER T., et al.: PET/CT for the staging and follow-up of patients with malignancies. European Journal of Radiology, 70 (3): 382-92, 2009.

8- PAVLIDIS N. and FIZAZI K.: Carcinoma of unknown primary (CUP). Crit. Rev. Oncol. Hematol., 69 (3): 2718, 2009.

9- ROH J.L., KIM J.S., LEE J.H., et al.: Utility of combined
${ }^{18}$ F-fluorodeoxyglucose-positron emission tomography and computed tomography in patients with cervical metastases from unknown primary tumors. Oral. Oncol., 45 (3): 218-24, 2009.

10- D'SOUZA M., JAIMINI A., BANSAL A., et al.: FDGPET/CT in Lymphoma. Indian J. Radiol Imaging, 23 (4): 354-65, 2013.

11- TAN G.J., BERLANGIERI S.U., LEE S.T., et al.: FDG PET/CT in the liver: Lesions mimicking malignancies. Abdominal Imaging Journal, 39 (1): 187-95, 2014.

12- De GAETANO A.M., RUFINI V., CASTALDI P., et al.: Clinical applications of ${ }^{18}$ F-FDG PET in the management of hepatobiliary and pancreatic tumors. Abdominal Imaging, 37 (6): 983-1003, 2012.

13- RACHH S. and BASU S.: PET/CT in patients with liver lesions of different nature. Clin. Transl. Imaging, 2 (2): 139-55, 2014

14- NIEKEL M.C., BIPAT S. and STOKER J.: Diagnostic imaging of colorectal liver metastases with CT, MR imaging, FDG PET, and/or FDG PET/CT: A meta-analysis of prospective studies including patients who have not previously undergone treatment. Radiology, 257 (3): 674 84, 2010.

15- PETROWSKY H., WILDBRETT P., HUSARIK D.B., et al.: Impact of integrated positron emission tomography and computed tomography on staging and management of gallbladder cancer and cholangiocarcinoma. J. Hepatol., 45 (1): 43-50, 2006.

16- KIM J.Y., KIM M.H., LEE T.Y., et al.: Clinical role of ${ }^{8}$ F-FDG PET-CT in suspected and potentially operable cholangiocarcinoma: A prospective study compared with conventional imaging. Am. J. Gastroenterol., 103 (5): 1145-51, 2008

17- ALI S.A. and ABD ELKHALEK Y.I.: Value of integrated PET/CT in detection of hepatic metastatic deposits. The Egyptian Journal of Radiology and Nuclear Medicine, 47 (2): 459-65, 2016.

18- ORLACCHIO A., SCHILLACI C., FUSCO N., et al.: Role of PET/CT in the detection of liver metastases from colorectal cancer. Radiol. Med., 114 (4): 571-85, 2009. 


\section{تقييم دور البوزيترون المنبعث المدمج بالآثعة المقطعية فى إكتشاف وتمييزيز آفات الكبد الهبد البؤرية}

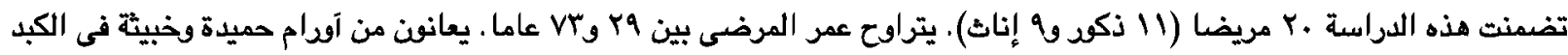

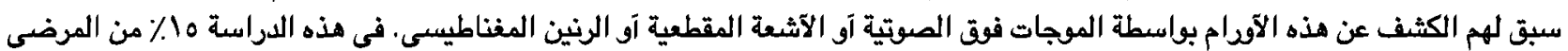

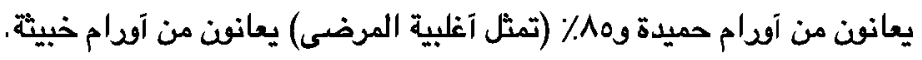

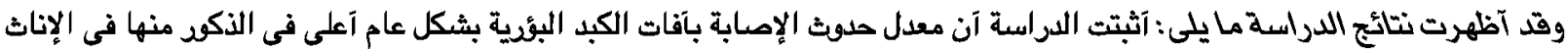

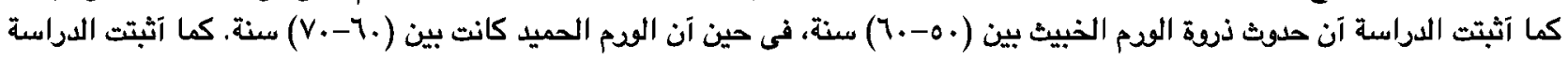

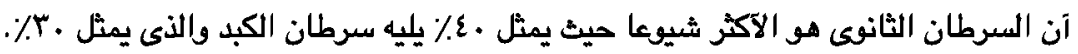

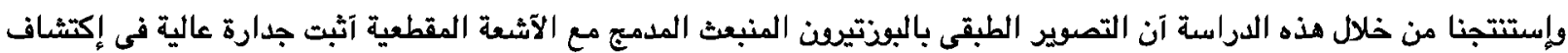

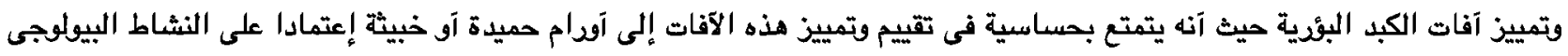
لهذه الآورام.

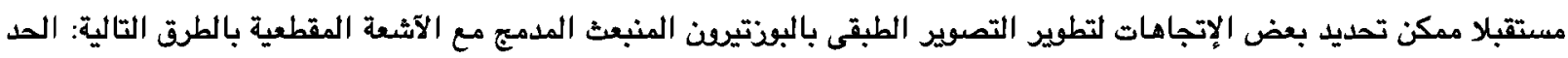

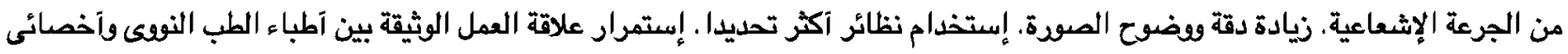

